\title{
Arterial hypertension in sub-Saharan Africa: analysis of the problem and treatment strategies
}

\author{
Marc Twagirumukiza \\ Supervisor: Lucas Van Bortel \\ Department of Pharmacology, Ghent University
}

Public defence: 15 April 2010

The present thesis contains both new evidences from personal surveys, as well as pooled information from existing scattered data about hypertension in sub-Saharan Africa (SSA). It has summarized useful data and put forward some aspects for hypertension management in this area. Specifically, it shows the present picture of the SSA region in terms of hypertension burden with high diversity between countries, and in terms of morbidity where the historical infectious diseases coexist nowadays with an increase of chronic diseases. The underlying reasons for this increase are multiple but dominated by modernization and lifestyle change leaded by socio-economic transition. Within the thesis, the quality of antihypertensive drugs has been analyzed (drug content, stability test and in vitro dissolution). Moreover the financial accessibility of hypertension treatment in SSA has been analyzed focusing on drug prices and usefulness of national essential medicines lists. Finally, the thesis suggests practical algorithms for hypertension management at community and health center levels in SSA. The author does not aim to cover all hypertension patterns in SSA but hopes to provide accurate and practical data for health professionals and/or decision-makers and to build up a basis for further research in the area.

The evidences from scattered studies done in SSA showed that besides poverty, malnutrition and infectious disease, there is an increase in hypertension prevalence. However limited data on the burden of hypertension in the SSA region are available. To manage the problem of hypertension in SSA affordable drugs of good quality should be accessible. Reports on the quality and affordability of antihypertensive drugs in SSA are scarce. Therefore this thesis documented the actual burden of hypertension. In addition the thesis documented the real market prices of anti-hypertensive drugs in SSA, and analysed the quality of these drugs, as well as the stability under tropical conditions storage.

Chapter one explains the physiopathology and epidemiology of hypertension in general and in sub-Saharan Africa in particular. Chapter two describes the outline of the thesis, and gives details on objectives, material and methods used in studies.

In chapter three the prevalence and burden of arterial hypertension in SSA is estimated. These estimates are based on 17 population studies from II SSA countries using the hypertensions definition of a blood pressure $\geq 140 / 90 \mathrm{mmHg}$, and conducted according the World Health Organization STEP-wise methodology (WHO STEPs) or similar 
approach. This study estimates the 2008 number of hypertensives (75 millions, CI95\% 65-93 million) more than 3 times higher than the last (2005) estimate of the World Health Organization Regional Office for Africa (20 millions), suggesting that the burden in SSA is underestimated. In addition this burden is expected to increase with $68 \%$ in the next 20 years. Reported prevalences of hypertension in SSA differ between countries (from $10.25 \%$ in Ethiopia to $23.0 \%$ in Ghana). Prevalences were consistently higher in urban than in rural settings (overall odds ratios between urban versus rural was $\mathbf{r}^{6} .5$, CI95\%: I.45-I.89). Population data on hypertension prevalence are incomplete or lacking in many countries underlining the need for national surveys.

Chapter four assessed the compliance of the existing (in 2007) National Essential Medicines Lists (NEMLs) with the World Health Organization/Essential Drug List (WHO/ EDL) and compares prices of antihypertensive drugs in and between 13 sub-Saharan African (SSA) countries. Data were collected on NEMLs and on drug prices from 65 public and 65 private pharmacies in 13 sub-Saharan countries ( 5 of each per country): Rwanda, Burundi, Tanzania, Uganda, DRC, Kenya, Cameroon, Congo, Gabon, Ivory Coast, Senegal, Niger and Mozambique. Prices from the market of every country were compared with prices indicated by the International Drug Price Indicator Guide (IDPIG). The cost of drug treatment within a country was calculated using defined daily doses (DDD) and between countries using DDD prices adjusted for Purchasing Power Parity Income per Capita. The chapter shows the existence of NEML in all surveyed countries, but also shows substantial differences in NEML composition between the I3 SSA countries. The proportion of NEMLs not regularly updated in the last 5 years (38\%) was double the global UN estimates. Prices of WHO/EDL advised drugs differ largely between drugs and for each drug within and between countries. In general, the use of drugs on the NEML had better financial accessibility and these drugs should be prescribed preferentially. Since hydrochlorothiazide is the cheapest drug, it should be the first drug to be considered.

In chapter five the quality of 17 formulations pertaining to 5 anti-hypertensive drugs, sampled in Rwandan pharmacies was investigated and the influence of tropical storage conditions was assessed. The drugs analysed are: atenolol, captopril, hydrochlorothiazide, methyldopa and propranolol. Drug content and in vitro dissolution were determined immediately after purchase (to) and after 6-months (t6) storage at room temperature and under simulated tropical conditions. For each drug, a brand formulation serving as reference was purchased in Belgium or France. The analysis shows that some antihypertensive drugs on the Rwandan market are not within standards from purchase on ( $20 \%$ of test formulations) and that the majority become substandard (for drug content and/or dissolution test) within 6 months storage under simulated tropical conditions ( $80 \%$ of test formulations). Hydrochlorothiazide test formulations showed the highest stability under tropical conditions. This shows the important influence of tropical climate conditions on drug formulations in Rwanda and probably also in other sub-Saharan African countries. This demonstrates the need for on-market drug quality monitoring.

In chapter six the main conclusions from the studies and management of hypertension in SSA are discussed. This showed that the international guidelines for management of hypertension are not fully applicable to the SSA setting. In this chapter an adapted algorithm for the management of hypertension in SSA countries is proposed. 